\title{
Cognitively driven brain machine control using neural signals in the parietal reach region
}

\author{
Eun Jung Hwang, and Richard A. Andersen
}

\begin{abstract}
This study demonstrates that the spiking and local field potential (LFP) activity in the parietal reach region (PRR) of the macaque monkey can be jointly used to control the location of the computer cursor when the correct target location must be inferred symbolically, e.g., leftward arrow for the leftward target, etc. The average correct target acquisition rate during this brain machine control task without actual movements was $86 \%$ for the six discrete target locations when using spikes and LFPs from 16 electrodes. This performance was significantly better than using spikes or LFPs alone. These results, together with our previous findings, suggest that a single decoder based on both spikes and LFPs in PRR can robustly provide the subjects' motor intent under varying contexts for neural prosthetic applications.
\end{abstract}

\section{INTRODUCTION}

$\mathrm{U}$ NTIL now neural prosthetics experiments have been limited to direct visuomotor mapping tasks in which the salient visual objects are the end-goals of the movement [15]. In real life, however, our movement goals are often formed in more complicated and cognitive ways than by direct visuomotor mapping [6]. For example, when playing musical instruments, we read the musical notes while performing a pre-learned cognitive mapping between the symbols and the movement goals. In a previous study, we found that the parietal reach region (PRR) in the posterior parietal cortex represents movement goals in a nearly identical manner for direct and symbolic visuomotor mappings [7], suggesting that PRR can serve as a source to decode the subjects' intended movement goals in both direct and cognitive-rule-based mappings for neural prosthetic applications.

Another aspect that has yet to be tested in neural prosthetics research is the benefits of using both spike and local field potential (LFP) signals jointly to decode the subject's motor intent [6, 8]. An increasing number of studies report that both spikes and LFPs encode critically relevant information for prosthetic applications such as movement target location, hand velocity, and hand grasp type [9-13]. However, cortical prosthetics experiments have been using either spikes or LFPs alone. Although some studies reported that LFPs and spikes can carry independent information, LFPs in general have been reported to be highly

Manuscript received April 1, 2010. This work was supported by National Institutes of Health under Grant EY 013337 and T32 NS007251.

E.J. Hwang is with California Institute of Technology, Pasadena, CA 91125 USA (corresponding author; phone: 626-395-8337; fax: 626-7952397; e-mail: eunjung@ caltech.edu).

R.A. Andersen is with California Institute of Technology, Pasadena, CA 91125 USA (e-mail: andersen@vis.caltech.edu). redundant and relatively unchanged across large distance $[6$, 13-18]. Thus, it remains unclear whether and how much the use of both spike and multi-site LFP signals can enhance the quality of decoding for prosthetic applications.

To explicitly address these two important questions, the feasibility of cognitively driven goal decoding and the benefits of the joint use of spikes and LFPs, we implemented a brain machine interface in which the movement goal was symbolically instructed, and the monkey's intended movement goal was decoded from both spikes and LFPs in PRR to control a cursor on the computer screen.

\section{PROCEDURE}

One male monkey (Macaca Mulatta, $8.3 \mathrm{~kg}$ ) participated in this study and all procedures followed National Institutes of Health guidelines.

\section{A. Behavioral experiment setup}

The monkey sat in a primate chair and viewed visual stimuli presented on an LCD monitor placed in the frontoparallel plane, $\sim 40 \mathrm{~cm}$ away from the eyes. The monkey's eye position was recorded with an infrared CCD camera (240 Hz; ISCAN, Burlington, MA) and hand position was recorded with a 19 inch translucent touch-sensitive screen (IntelliTouch; ELO Systems, Menlo Park, CA) placed against the LCD monitor. The visual stimulus presentation, online monitoring of eye and hand positions, and reward control were handled by a real-time LabView program (National Instruments, LabView7.1).

The monkey completed 15 experimental sessions each of which consisted of two sets: calibration and brain control. During the calibration set the monkey made actual reaches so that we could characterize how each of neural signals encoded the reach target location. During the brain control set we decoded the monkey's intended target location using the neural signals and their encoding properties in order to control the computer cursor without the monkey actually reaching. The details of each set are below.

\section{B. Calibration set}

The monkey began a trial by acquiring the ocular and manual fixations at the screen center (Fig. 1A). After a $0.5 \mathrm{~s}$ fixation period, an arrow (symbol) appeared in the center for $0.3 \mathrm{~s}$ and a variable delay $(1.2 \pm 0.15 \mathrm{~s})$ followed. The monkey maintained the fixations until the manual fixation target disappeared (go-cue), signaling the monkey to initiate a reach without moving the eyes (reaction time $=0.3 \pm 0.13$ s). Once the reach ended on the touch screen and was within $3^{\circ}$ of any one of the six invisible targets, a green circle 
appeared at the target that was the closest to the reach endlocation. The reach trials that ended outside $3^{\circ}$ were aborted. If the closest target was correct, the monkey received a juice reward after holding the hand on the reach end-location and eyes on the fixation target for $0.3 \mathrm{~s}$. The six invisible targets were evenly spaced around a virtual circle (10.3 ${ }^{\circ}$ eccentricity) and the one positioned in the pointing direction of the arrow was the correct target. The six arrow directions were pseudo-randomly interleaved. The monkeys made 20 successful trials per arrow direction.
A

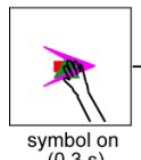
symbol on
$(0.3 \mathrm{~s})$

B

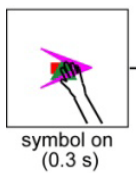

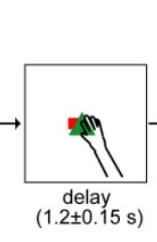

Calibration set

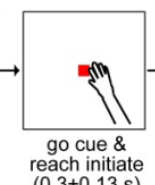

$(0.3 \pm 0.13 \mathrm{~s})$

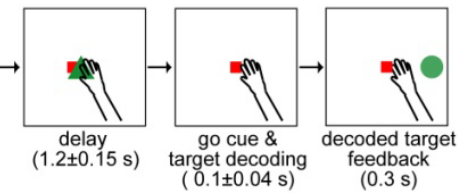

Brain control set

Fig. 1. Task sequence for the calibration and brain control sets.

\section{Brain control set}

The brain control trial was the same as the calibration trial until the go-cue onset (Fig. 1B). Concurrently with the gocue, the monkey's intended target location was decoded based on the neural signals during the latest $1 \mathrm{~s}$ and then a green circle appeared at the decoded target location that was one of the six invisible targets. If the decoded target matched the arrow direction, the monkey received a juice reward after maintaining the manual and ocular fixations in the center for another $0.3 \mathrm{~s}$. A brain control set included 176 to 894 trials $(534 \pm 183.4)$.

\section{Neural recording}

The monkey was implanted with a head holder and a recording chamber housing a 16-channel chronic microdrive (Neuralynx, Bowsman, MT) following the procedures described in Hwang and Andersen [19]. Sixteen electrodes were strategically placed over the intra parietal sulcus (IPS) guided by structural magnetic resonance imaging so that most of them would be located in PRR, i.e., medial bank of IPS. The electrodes were spread over a $2 \times 6.5 \mathrm{~mm}^{2}$ area along the IPS on the brain surface but they became more densely populated once they entered the brain due to oblique incidence angles to hit IPS in the center of chamber. Across 15 sessions, the electrode locations were adjusted daily in depth by small amounts to improve the quality of unit isolations. A commercial sixteen channel neural signal recording system (Plexon MAP, Dallas, TX) was used to record and store neural signals. LFPs and spikes were separated by the hardware band-pass filters in a preamplifier (LFP: $3.3-88 \mathrm{~Hz}$ and spike: $154 \mathrm{~Hz}-8.8 \mathrm{kHz}$ ). We performed online spike detection and sorting using a commercial software package (Plexon Rasputin). The spike count and LFP power computation, and target decoding were done using the customized real time MATLAB code.

\section{E. Decoding algorithm}

To decode the intended target location, a Bayesian classifier with a uniform prior probability distribution was implemented. For each channel, the spike events and the LFP signal for the last $1 \mathrm{~s}$ interval of the delay period were processed for decoding. Each channel could have up to 3 units and their spike counts were computed. The LFP signal of each channel was subjected to FFT and reduced to the average power in 10 evenly divided frequency bands (0-10 $\mathrm{Hz}, 10-20 \mathrm{~Hz}, \ldots, 90-100 \mathrm{~Hz})$. Therefore, the total dimensions of the observation data could be up to 208, i.e., 16 channels $\times(3$ spike counts +10 LFP power $) /$ channel. However, we reduced the data dimensions by selecting those dimensions that were significantly tuned to the target location during the calibration set (one way ANOVA with a factor for target location, $\mathrm{p}<1 \mathrm{e}-3$ for spikes and $\mathrm{p}<1 \mathrm{e}-23$ for LFPs). On average, each session had 16 significantly tuned dimensions $(16.1 \pm 5.47) ; 7.6( \pm 2.03)$ units and $8.5( \pm 5.08)$ LFP bands. Under the assumption that the data in these selected dimensions were normally distributed, we estimated the mean and covariance matrices for each of six target locations using the calibration set trials. Since we also assumed that covariance remains the same across all targets, the six covariance matrices were averaged to produce a single covariance used for all targets. During the brain control set, the posterior probability of the given observation was computed for each of six target locations using the estimated mean and covariance and the target with the maximum probability was selected as the monkey's intended target location.

\section{RESULTS}

Fig. 2 displays the spatial tuning of 17 neural signals to the reach target location during the calibration and brain control sets in a typical session. Seven of them were spike counts and the rest were LFP power, mostly in the $40-50 \mathrm{~Hz}$ band. These neural signals were selected because their delay period activity was significantly tuned to the impending reach target location during the calibration set. For the brain control set, all trials, correct or not, were included to compute the tuning. Similar tuning curves between the two sets are necessary to achieve high performance in the brain control set. Thus, we examined tuning similarity between the two sets for all features used in 15 sessions. First, to compare the shape of the two tuning curves for each feature, we computed the Pearson's correlation coefficient between the two curves. The correlation coefficient was $0.93 \pm 0.154$ on average, indicating that the shape was conserved between the two sets. Second, we measured the trough-to-peak amplitude of each tuning curve as its tuning depth and computed the difference in the tuning depth between the calibration and brain control sets for each feature. Since different neural signals have different dynamic ranges, we normalized the tuning depth difference as a percent ratio to the peak amplitude of the calibration tuning curve for each feature. The normalized difference in tuning depth between the two sets was $12 \pm 16.5 \%$ on average. The tuning depth 
was slightly smaller during the brain control set than the calibration set. Nevertheless, the overall difference was small enough to produce successful target decoding for most trials in the brain control set as shown hereafter.

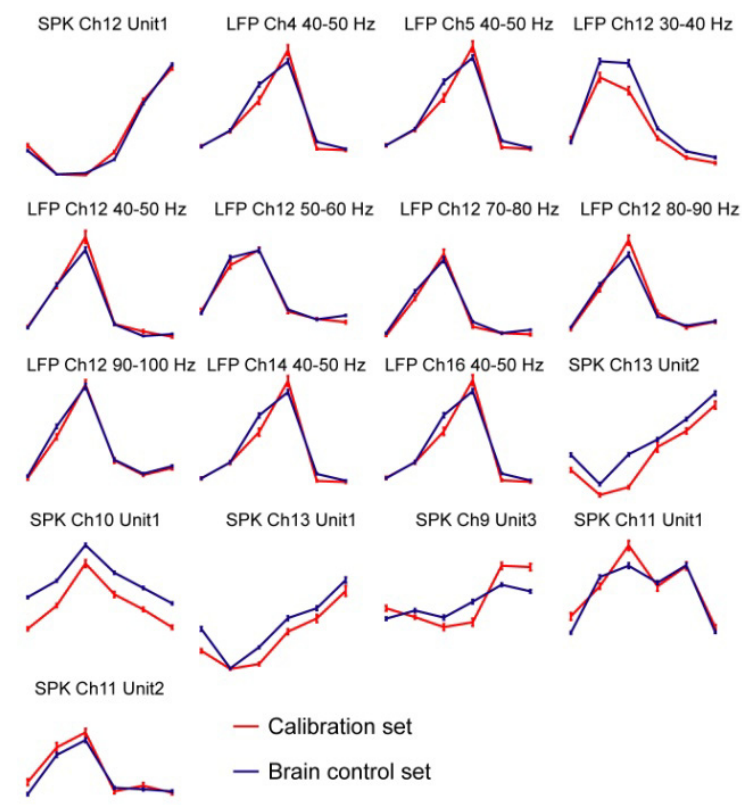

Fig. 2. The spatial tuning of example neural signals during the calibration and brain control sets of a typical session. In each panel, the 6 different target locations are on the x-axis and the spike count or LFP power is on the y-axis. The mean and S.E.M are shown.

Fig. 3A shows the success rate in the brain control set conducted using the neural signals shown in Fig. 2. The success rate was computed for every 60 consecutive trials. Throughout the set, the success rate maintained about $85 \%$. Most of errors occurred because the decoder misclassified the immediate neighbors of the correct target as seen in the confusion matrix in Fig. 3B. To examine the performance enhancement achieved by the use of both signals, we implemented offline decoders using only spike counts or LFP power (Fig. 3C and D). The decoding accuracy was $69 \%$ using spikes alone and 58\% using LFPs alone, each of which was worse than using both signals together $(85 \%)$. The confusion matrices indicate that the spikes were poor at decoding the first two targets whereas the LFPs were poor at decoding the last two targets. Therefore, combining these two signals compensated for the weakness of each signal, producing higher decoding performance overall.

Fig. 4 shows the within-session peak performance, the maximum success rate over sliding 60 consecutive trials, in all sessions when using both signals, spikes only, and LFPs only. The performance during the actual brain control sets corresponds to the success rate computed using both signals. The mean success rate was $86 \pm 4.5 \%, 76 \pm 6.0 \%$, and $59 \pm 10.9 \%$ respectively for the three cases. Therefore, the correct target decoding rate was higher when using both signals than using spikes alone (paired t-test; $\mathrm{p}<1 \mathrm{e}-6$ ) or using LFPs alone (paired t-test; $\mathrm{p}<2 \mathrm{e}-8$ ). Correct target decoding was better when using only spikes than using only LFPs (paired t-test; $\mathrm{p}<2 \mathrm{e}-5$ ), consistent with a previous finding [9]. Interestingly, the performance when using both

signals varied less across sessions than the performance when using either signal by itself (S.D. is 4.0, 6.0, and $10.9 \%$ respectively for decoding from both, spikes and LFPs). This would be an additional benefit of using both signals, preventing a steep performance degradation caused by the loss of spikes or noise in LFPs as shown on sessions $5,6,8,10$ and 15 .

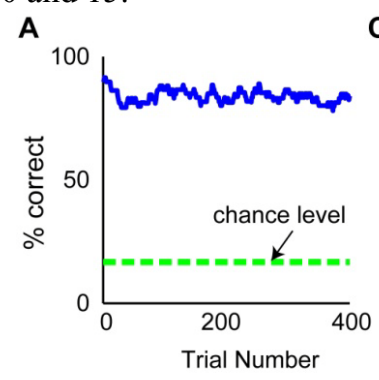

C

B

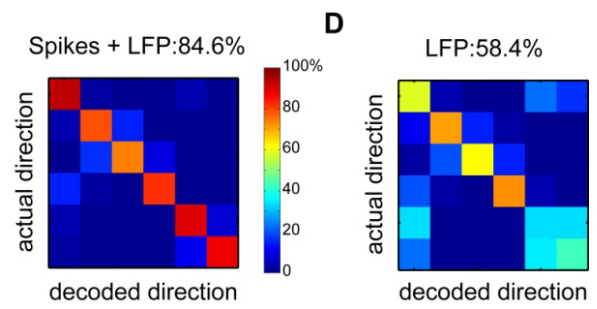

Fig. 3. Brain control task performance in a typical session. A. Percent correct for every 60 consecutive trials. B. Confusion matrix of the actual decoder used in the brain control set. The color code shows the relative number of times that a presentation of target $i$ (on the y-axis) was decoded to be target $\mathrm{j}$ (on the $\mathrm{x}$-axis). C-D. Confusion matrices of the offline decoder using spikes or LFPs by themselves.

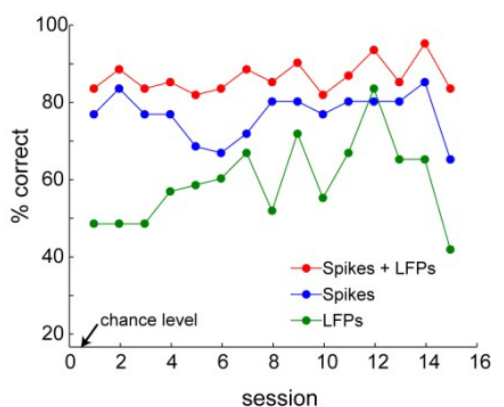

\section{DISCUSSION}

Here, we showed that the neural signals in PRR can be harnessed to control a computer cursor even in a highly cognitive context. These results are consistent with our previous findings that PRR neuronal activity near reach onset encodes the reach target in a task-invariant manner for both direct and symbolically instructed reach tasks, suggesting that PRR can serve as a universal neural substrate to provide control signals for the neural prosthetics under various contexts [7]. Moreover, the use of both spikes and LFPs for decoding significantly boosted the brain control task performance and made the performance less variable across sessions compared to the use of either signal by itself.

However, the performance boost reported here should not be taken as the best achievable for several reasons. First, we used a very simplistic feature selection method. That is, the spike counts from the sixteen channels were stacked on top of the LFP power in the ten frequency bands from each of 
the sixteen channels and then only the dimensions that were significantly correlated with the reach target during the calibration set were selected. Although this correlation criterion may approximate an effective feature selection, in general it does not maximize the predictive power of the given data. For example, even features that are not correlated with the predicted variable by themselves could have a significant predictive power when combined together [20]. Thus, other methods of feature selection including other filter, wrapper, or embedded methods are worthwhile to explore [20,21]. Second, although the spike counts and LFP power in PRR are known to carry information about the target location, other aspects in these signals, e.g., the phase of LFP signal or spike-field coherence, have not been fully investigated yet. Thus, more optimal or additionally informative features may be found in future studies. Third, our assumption that the spike counts and the LFP power are normally distributed does not hold true in general. For example, spike counts follow a Poisson distribution rather than a normal distribution [22], thus approximation using normal distributions could be particularly detrimental when the mean is low. Moreover, the log power instead of the raw LFP power would better follow normality given that the power is a positive quantity [23]. Finally, our decoding algorithm was a simple Bayesian classifier. Many other decoding algorithms, such as nearest-neighbor, Fisher linear discriminant, or support vector machine, trained artificial neural-networks, may outperform the simple classifier used in our study [8]. Taken together, future studies are warranted to explore ways to further enhance the decoding performance when using both spikes and LFPs.

\section{ACKNOWLEDGMENT}

We thank K. Pejsa and N. Sammons for animal care, and V. Shcherbatyuk and T. Yao for technical and administrative assistance.

\section{REFERENCES}

[1] C. T. Moritz, S. I. Perlmutter, AND E. E. Fetz, "Direct Control OF PARALYSED MUSCLES BY CORTICAL NEURONS," NATURE, VOL. 456, PP. 639-642, 2008.

[2] L. R. Hochberg, M. D. Serruya, G. M. Friehs, J. A. Mukand, M. Saleh, A. H. Caplan, A. Branner, D. Chen, R. D. PenN, and J. P. DONOGHUE, "NEURONAL ENSEMBLE CONTROL OF PROSTHETIC DEVICES BY A HUMAN WITH TETRAPLEGIA," NATURE, VOL. 442, PP. 164-71, 2006.

[3] S. Musallam, B. D. Corneil, B. Greger, H. Scherberger, and R. A. ANDERSEN, "COGNITIVE CONTROL SIGNALS FOR NEURAL PROSTHETICS," SCIENCE, VOL. 305, PP. 258-262, 2004.

[4] M. Velliste, S. Perel, M. C. Spalding, A. S. Whitford, and A. B. SCHWARTZ, "CORTICAL CONTROL OF A PROSTHETIC ARM FOR SELFFEEDING," NATURE, 2008.

[5] J. Wessberg, C. R. Stambaugh, J. D. Kralik, P. D. Beck, M. LaUbach, J. K. Chapin, J. Kim, S. J. BigGS, M. A. SRinivasan, AND M. A. NICOLELIS, "REAL-TIME PREDICTION OF HAND TRAJECTORY BY ENSEMBLES OF CORTICAL NEURONS IN PRIMATES," NATURE, VOL. 408, PP. 361-5, 2000.

[6] R. A. Andersen, E. J. Hwang, and G. H. Mulliken, "Cognitive NEURAL PROSTHETICS," ANNU REV PSYCHOL, VOL. 61, PP. 169-90, C1$3,2010$.

[7] E. J. Hwang and R. A. ANDERSEN, "THE PARIETAl REACH REgion REPRESENTS THE SPATIAL GOAL IN SYMBOLICALLY INSTRUCTED
REACHES," PRESENTED AT SOCIETY FOR NEUROSCIENCE, WASHINGTON DC, 2008.

[8] R. QUiAn Quiroga and S. PANZERI, "EXTRACTING INFORMATION FROM NEURONAL POPULATIONS: INFORMATION THEORY AND DECODING APPROACHES," NAT REV NEUROSCI, VOL. 10, PP. 173-185, 2009.

[9] H. SCherberger, M. R. Jarvis, AND R. A. ANDERSEn, "Cortical LOCAL FIELD POTENTIAL ENCODES MOVEMENT INTENTIONS IN THE POSTERIOR PARIETAL CORTEX," NEURON, VOL. 46, PP. 347-354, 2005.

[10] D. A. Heldman, W. WANG, S. S. Chan, AND D. W. Moran, "LOCAL FIELD POTENTIAL SPECTRAL TUNING IN MOTOR CORTEX DURING REACHING," IEEE TRANS NEURAL SYST REHABIL ENG, VOL. 14, PP. 1803, 2006.

[11] I. Asher, E. Stark, M. Abeles, and Y. Prut, "Comparison of DIRECTION AND OBJECT SELECTIVITY OF LOCAL FIELD POTENTIALS AND SINGLE UNITS IN MACAQUE POSTERIOR PARIETAL CORTEX DURING PREHENSION," J NEUROPHYSIOL, VOL. 97, PP. 3684-95, 2007.

[12] C. Mehring, J. Rickert, E. VAadia, S. C. DE Oliveira, A. Aertsen, AND S. ROTTER, "INFERENCE OF HAND MOVEMENTS FROM LOCAL FIELD POTENTIALS IN MONKEY MOTOR CORTEX," NAT NEUROSCI, VOL. 6, PP. 1253-1254, 2003.

[13] R. L. Spinks, A. Kraskov, T. Brochier, M. A. Umilta, AND R. N. LEMON, "SELECTIVITY FOR GRASP IN LOCAL FIELD POTENTIAL AND SINGLE NEURON ACTIVITY RECORDED SIMULTANEOUSLY FROM M1 AND F5 IN THE AWAKE MACAQUE MONKEY," J NEUROSCI, VOL. 28, PP. 10961-71, 2008

[14] J. G. O'Leary and N. G. Hatsopoulos, "EARly visuomotor REPRESENTATIONS REVEALED FROM EVOKED LOCAL FIELD POTENTIALS IN MOTOR AND PREMOTOR CORTICAL AREAS," $J$ NEUROPHYSIOL, VOL. 96, PP. 1492-1506, 2006.

[15] K. D. Harris, D. A. Henze, H. Hirase, X. Leinekugel, G. Dragoi, A. CZURKo, AND G. BUZSAKI, "SPIKE TRAIN DYNAMICS PREDICTS THETA-RELATED PHASE PRECESSION IN HIPPOCAMPAL PYRAMIDAL CELLS," NATURE, VOL. 417, PP. 738-741, 2002.

[16] M. Saleh, J. Reimer, R. Penn, C. L. Ojakangas, and N. G. HATSOPOULOS, "FAST AND SLOW OSCILLATIONS IN HUMAN PRIMARY MOTOR CORTEX PREDICT ONCOMING BEHAVIORALLY RELEVANT CUES," NEURON, VOL. 65, PP. 461-471, 2010.

[17] P. Berens, G. A. Keliris, A. S. Ecker, N. K. Logothetis, AND A. S TOLIAS, "FEATURE SELECTIVITY OF THE GAMMA-BAND OF THE LOCAL FIELD POTENTIAL IN PRIMATE PRIMARY VISUAL CORTEX," FRONT NEUROSCI, VOL. 2, PP. 199-207, 2008.

[18] A. Belitski, A. Gretton, C. Magri, Y. Murayama, M. A Montemurro, N. K. LOgothetis, and S. PANZERI, "LowFREQUENCY LOCAL FIELD POTENTIALS AND SPIKES IN PRIMARY VISUAL CORTEX CONVEY INDEPENDENT VISUAL INFORMATION," $J$ NEUROSCI, VOL. 28, PP. 5696-709, 2008.

[19] E. J. HWANG AND R. A. ANDERSEN, "Brain CONTROL OF MOVEMENT EXECUTION ONSET USING LOCAL FIELD POTENTIALS IN POSTERIOR PARIETAL CORTEX," J NEUROSCI, VOL. 29, PP. 14363-70, 2009.

[20] I. GUYON AND A. ELISSEEFF, "AN INTRODUCTION TO VARIABLE AND FEATURE SELECTION " JOURNAL OF MACHINE LEARNING RESEARCH, VOL. 3, PP. 1157-1182, 2003.

[21] W. Zhisong, A. Maier, N. K. Logothetis, and L. Hualou, "RELAXATION-BASED FEATURE SELECTION FOR SINGLE-TRIAL DECODING OF BISTABLE PERCEPTION," BIOMEDICAL ENGINEERING, IEEE TRANSACTIONS ON, VOL. 56, PP. 101-110, 2009.

[22] P. DAYAN AND L. F. ABBOtT, THEORETICAL NEUROSCIENCE: COMPUTATIONAL AND MATHEMATICAL MODELING OF NEURAL SYSTEMS: MIT PRESS, 2001.

[23] E. L. CROW AND K. Shimizu, LOGNORMaL Distributions: theORY AND APPLICATIONS. NEW YORK, NY: MARCEL DEKKER, INC., 1988. 